\title{
Passive Phase Conjugation Applied to An OFDM Based Terrestrial Communication System
}

\author{
Pei $\mathrm{Xiao}^{\dagger}$, Senior Member, IEEE, Vincent Fusco ${ }^{\dagger \dagger}$, Fellow, IEEE
}

\begin{abstract}
The demand for high data rate radio systems that can operate robustly in multipath environments presents challenging technical issues. In this paper, we propose a Passive Phase Conjugation (PPC) based Orthogonal Frequency Division Multiplexing (OFDM) system. Compared to the conventional OFDM system, the proposed system achieves a lower computational complexity since the need for conventional channel estimation and equalisation can be eliminated. The performance gain of the proposed technique is shown to be particularly advantageous in systems with multiple receiver array elements. Numerical results are provided in order to illustrate the performance of the system when operating through a multipath radio channel. We also derive a closed form formula which can be used to predict the performance of the proposed scheme over multipath channels with uniform power delay profile. Our analysis validates the simulation results and provides insights into the behavior of wireless centric OFDM-PPC systems.
\end{abstract}

\section{INTRODUCTION}

In a wireless radio communication system a transmitted signal will follow many different propagation paths before arriving at the receiver. This causes intersymbol interference (ISI) and degrades system performance. A similar situation arises in underwater acoustic communication systems, such as between remotely operated surveillance vehicles, underwater sensor networks, etc. The shallow water acoustic channel is particularly problematic as it exhibits a large amount of multipath interference. Both radio and acoustic scenarios give rise to a frequency selective fading channel which impairs high data rate transmission. Various solutions have been proposed to improve transmission data rate and reliability in both radio and shallow water acoustic channels. Coherent receivers that use adaptive equalisation have been proposed, e.g., in [1], [2] to combat the ISI caused by frequency selective channels. However, a large number of taps are usually required by the equalisers for dispersive channels and these taps must be constantly adjusted to adopt to the changing environment, resulting in a substantial computational burden. Other solutions have also been proposed, e.g., direct sequence spread spectrum modems coupled with matching pursuit algorithms [3] as well as passive phase conjugation (PPC) [4], [5], which provides a simple low data rate receiver solution.

Copyright (c) 2012 IEEE. Personal use of this material is permitted. However, permission to use this material for any other purposes must be obtained from the IEEE by sending a request to pubs-permissions@ieee.org. The author ${ }^{\dagger}$ is with the Centre for Communication Systems Research (CCSR), University of Surrey, Guildford, Surrey, GU2 7XH, United Kingdom. (e-mail: pei.xiaodsurrey.ac.uk). The author ${ }^{\dagger \dagger}$ is with the Institute of Electronics, Communications and Information Technology (ECIT), Queen's University Belfast, BT3 9DT, Northern Ireland. (e-mail: v.fuscodecit.qub.ac.uk).

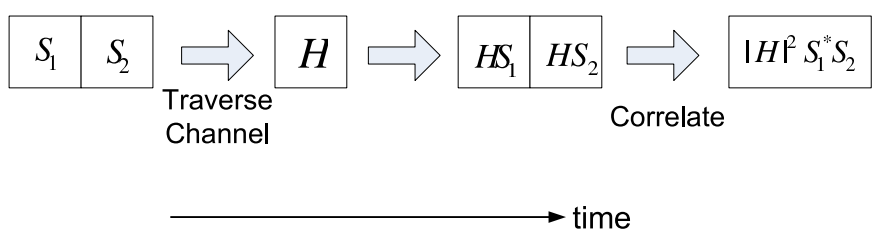

Fig. 1. Passive Phase Conjugation.

The PPC procedure [6] shown in Fig. 1 begins with a source transmitting a signal $S_{1}$ used as a single probe. After waiting for the multipath arrivals to clear, the source then transmits the data stream $S_{2}$. At each element in a distant receiving array, the received probe is cross-correlated with the received data stream. This cross-correlation is done in parallel at each array element and the results are summed across the array to obtain the signal suitable for demodulation. As the propagation medium changes it becomes necessary to break up the data stream and insert a new probe. This scheme relies on pulse position or DPSK modulation for probe and data encoding [6], and multiple chips are required to carry a single information bearing signal, leading to low bit rates. Variants of the PPC technique have been proposed to recover the data rate loss as a result of probe retransmission, for example, a decision-directed phase-conjugation approach was proposed in [7] where the detected data stream was used to regenerate the channel estimates. Adaptive channel estimation was proposed in [8], [9] to generate the up-to-date timereversed filters directly from the received information-bearing signal, thus eliminating the need for probe retransmission. In this case, the increase in data transmission rate comes at the expense of increased computational complexity. For simplicity, we use the probe based approach and achieve a complexity reduction at the cost of a decrease in data rate due to the probe transmission.

Both active phase conjugation and passive phase conjugation have been demonstrated using experimental data collected in shallow water in [10], [11] and [4], [12], respectively, for two-way and one-way communications. The phase conjugation technique has been further extended to multipleinput, multiple-output (MIMO) communications in [13], [14]. Active phase conjugation (APC) and time reversal techniques have also been applied to radio channels in [15], [16]. An overview of the previous experimental and analytical work on PPC can be found in [17].

Orthogonal frequency division multiplexing (OFDM) [18] is well suited for broadband applications since it effectively 
transforms a frequency selective channel into parallel flat fading channels, which simplifies both channel estimation and data recovery at receiver. The OFDM technique has been adopted in several wireless standards, e.g., 802.11a wireless local network (WLAN) system [19], and 3GPP Long Term Evolution (LTE), also known as Evolved-UMTS Terrestrial Radio Access [20], [21]. The OFDM technique has also been previously proposed to transmit data in the shallow water acoustic channel in [22]-[24]. In particular, OFDM and PPC have been jointly applied to underwater communications in [23], [24], where the conventional channel estimation and equalisation are employed to detect transmitted signals in a coherent manner.

In this paper, a hybrid OFDM-PPC method is developed with potential application to terrestrail wireless communications. The proposed OFDM-PPC scheme exploits desirable properties of both OFDM and PPC to create a very effective communication system. Like conventional PPC, APC and time reversal methods, the proposed OFDM-PPC scheme removes the need for explicit channel estimation and subsequent equalisation, it delivers high performance data transfer while requiring only a minor modification to the conventional OFDM wireless receiver architecture.

The remainder of this paper is organized as follows. In Section II, we briefly review the structure of the conventional OFDM system. In Section III, we propose a PPC based OFDM system with multiple array elements. Simulation results are presented in Section IV to demonstrate the effectiveness of the proposed scheme. In Section V, the performance of the proposed OFDM-PPC scheme is analyzed theoretically and compared with simulation results for multipath channels with uniform power delay profile. Finally, conclusions are drawn in Section VI.

\section{Conventional OFDM System}

Fig. 2 shows the block diagram of a classic OFDM system [18]. At the transmitter, each group of two information bits $\left\{c_{n}\right\}$ is mapped into one of four QPSK symbols $s_{n}$. A set of $N$ symbols is serial to parallel converted and imposed onto orthogonal sub-carriers by means of the inverse fast Fourier transform (IFFT). The output from IFFT block is then converted into serial data and a cyclic prefix (CP) is inserted to form one OFDM symbol for transmission. The length of the $\mathrm{CP}$ is assumed to be longer than the impulse response of the channel in order to combat inter-symbol interference and inter-carrier interference. The use of a cyclic prefix longer than the channel delay spread will transform the linear convolution in the channel to a cyclic convolution.

After CP removal and FFT operation, the received signal becomes

$$
y_{n}=s_{n} \cdot H_{n}+\eta_{n},
$$

where $\left\{y_{n}\right\}$ contains $N$ received data samples, $\left\{s_{n}\right\}$ contains $N$ transmitted symbols; $\eta_{n}$ represents uncorrelated Gaussian noise with zero mean and variance $\sigma_{\eta}^{2}$, i.e., $\eta_{n} \sim \mathcal{C N}\left(0, \sigma_{\eta}^{2}\right)$; $H_{n}$ is the frequency response of the channel at the $n$th subcarrier.
In conventional OFDM systems, either a one-tap zeroforcing (ZF) or minimum mean square error (MMSE) equaliser is applied to detect the transmitted symbol at the receiver, i.e., $\hat{s}_{n}=\alpha_{n} y_{n}$, the equaliser coefficient can be derived as [18]

$$
\alpha_{n}=\left\{\begin{array}{lr}
1 / \hat{H}_{n}, & \text { ZF equaliser } \\
\frac{\hat{H}_{n}^{*}}{\left|\hat{H}_{n}\right|^{2}+\sigma_{v}^{2}}, & \text { MMSE equaliser }
\end{array}\right.
$$

where $(\cdot)^{*}$ denotes the conjugate operation, $\hat{H}_{n}$ is an estimate of $H_{n}$, and $n=1,2, \ldots, N$. In order to perform equalisation, we need to obtain an estimate of the channel frequency response $H_{n}$ for each subcarrier by some channel estimation algorithm.

\section{Passive Phase Conjugate Based OFDM System}

Passive phase conjugation removes the need for explicit recovery of the channel and its subsequent equalisation. This follows from the fact that PPC implicitly recombines the multipath arrival signals instead of trying to invert the channel as required by direct channel equalisation methods. Fig. 1 illustrates the principle of PPC which operates as follows [6]: the transmitter sends signals $S_{1}$ and $S_{2}$ in sequence. These signals traverse the channel $H$, which is assumed to be static over the transmission period of $S_{1}$ and $S_{2}$, and are observed by the receiver as $H S_{1}$ and $H S_{2}$; the receiver cross-correlates $H S_{1}$ and $H S_{2}$, producing $|H|^{2} S_{1}^{*} S_{2}$. The autocorrelation of the channel impulse response $|H|^{2}$ acts to reconcentrate, i.e., to focus coherently, the multipath arrivals. Note that channel variations between $S_{1}$ and $S_{2}$ would degrade the performance of PPC due to imperfect focusing.

However, PPC cannot completely eliminate ISI due to the sidelobes in the channel autocorrelation function after focusing, which entails the use of equalisation technique. Even so, unlike the system without phase conjugation only simple equalisers are needed due to the focusing effect for the multipath arrivals. Note that a rich scattering environment enhances the coherent focusing effect because in this case, the channel coefficients appear noise-like, resulting in an autocorrelation that has a strong peak at the zero lag and is nearly zero at all other lags. An antenna array positioned at the receiver facilitates even better temporal focusing since upon averaging, all elements contribute to the same main peak, but spread the autocorrelation sidelobes [25].

In the PPC system employed in [6], the message is encoded within the correlation of the two consecutively transmitted waveforms $S_{1}$ and $S_{2}$. To this end, two signalling schemes, namely, pulse position modulation (PPM) and differential phase shift keying (DPSK) with Gold sequences were proposed [6]. The major shortcoming of these schemes is low spectral efficiency since multiple chips are required to represent a single information symbol. Furthermore, the length of the chip sequence has to be large in order to achieve good cross-correlation properties. There are some alternative PPC techniques, e.g., one can first estimate the channel and then correlate with the data stream, and the channel estimate is updated as the environment changes. 


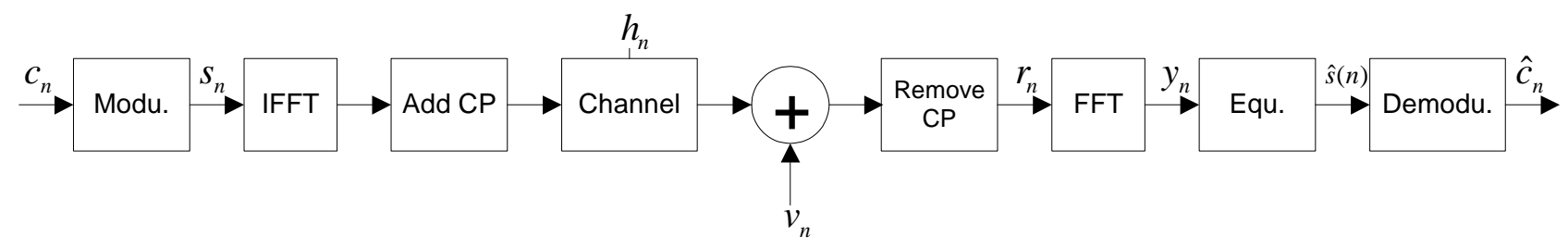

Fig. 2. Structure of a conventional single channel OFDM system.

In this paper, we propose a PPC based OFDM system which significantly outperforms the PPC system mentioned above, and has additional benefits with respect to the complexity compared to the conventional OFDM system.

Fig. 3 shows the proposed system with a single source and $N_{r}$ receiver array elements corresponding to $N_{r}$ indepdendent FFT-PPC chains at the receiver side. The transmitter has exactly the same structure as in the conventional OFDM system. Each FFT-PPC chain at the receiver is also similar to the conventional OFDM system, but notably the equaliser block is replaced by a PPC block. The operation of the revised system is now elaborated.

In the proposed OFDM-PPC system, $S_{1}$ is represented by a probe sequence $\mathbf{p}=\left[\begin{array}{llll}p_{1} & p_{2} & \ldots & p_{N}\end{array}\right]$; and $S_{2}$ is represented by the transmitted symbol sequence $\mathbf{s}=$ $\left[\begin{array}{llll}s_{1} & s_{2} & \ldots & s_{N}\end{array}\right]$. The elements of $\mathbf{p}$ are chosen to be real positive scalars, i.e., they do not introduce any phase shifts.

The inputs of the $k$ th PPC corresponding to the probe $\mathbf{p}$ and data $\mathbf{s}$ are

$$
\begin{aligned}
\mathbf{y}_{\mathbf{p}}(k) & =\left[\begin{array}{lll}
p_{1} H_{1}(k)+\epsilon_{1}(k) & \ldots & p_{N} H_{N}(k)+\epsilon_{N}(k)
\end{array}\right] ; \\
\mathbf{y}_{\mathbf{s}}(k) & =\left[\begin{array}{lll}
s_{1} H_{1}(k)+\xi_{1}(k) & \ldots & s_{N} H_{N}(k)+\xi_{N}(k)
\end{array}\right],
\end{aligned}
$$

where $\epsilon_{n}(k)$ and $\xi_{n}(k)$ are zero-mean additive white Gaussian noise with variance $N_{0}$, i.e., $\epsilon_{n}(k), \xi_{n}(k) \sim \mathcal{C N}\left(0, N_{0}\right)$; $H_{m}(k)$ is the frequency response of the $m$ th subcarrier corresponding to the channel between the source and the $k$ th $\left(k=1,2, \ldots, N_{r}\right)$ receiver array element. Performing phase conjugation (element-wise cross-correlation of the above two sequences) and summing up the phase conjugation outputs of individual branches, we obtain

$$
\mathbf{z}=\left[\begin{array}{c}
p_{1} s_{1} \sum_{k=1}^{N_{r}}\left|H_{1}(k)\right|^{2}+\omega_{1} \\
\vdots \\
p_{N} s_{N} \sum_{k=1}^{N_{r}}\left|H_{N}(k)\right|^{2}+\omega_{N}
\end{array}\right]^{T},
$$

where $(\cdot)^{T}$ denotes transpose operation, the noise term $\omega_{n}=$ $\sum_{k=1}^{N_{r}} \omega_{n}(k)$, and

$$
\begin{aligned}
\omega_{n}(k) & =\epsilon_{n}^{*}(k) s_{n} H_{n}(k)+\xi_{n}(k) p_{n} H_{n}^{*}(k)+\epsilon_{n}^{*}(k) \xi_{n}(k) ; \\
n & =1, \ldots, N .
\end{aligned}
$$

Equation (4) holds since the elements of $\mathbf{p}$ are chosen to be real positive scalars, e.g., $p_{n}^{*}=p_{n}$. Equation (4) shows
TABLE I

COMPLEXITY COMPARISON BETWEEN OFDM (WITH MMSE EQUALISATION) AND OFDM-PPC. $N$ IS THE FFT SIZE, $N_{r}$ IS THE NUMBER OF ARRAY ELEMENTS IN THE RECEIVER, $M$ IS THE NUMBER OF OFDM SYMBOLS TRANSMITTED AFTER EACH PROBE/PILOT SYMBOL.

\begin{tabular}{|c|c|c|c|}
\hline \hline operations & $\div$ & $\times$ & + \\
\hline OFDM & $2 N N_{r}$ & $N N_{r}(M+1)$ & $N N_{r} M$ \\
\hline OFDM-PPC & 0 & $N N_{r} M$ & $N N_{r}(M-1)$ \\
\hline
\end{tabular}

that the multipath induced phase shifts have been removed by the passive phase conjugation operation. Consequently, the transmitted symbols can be recovered without the need for channel estimation (using pilot carriers) and subsequent channel equalisation. It should be noted that with the scheme introduced in [23], [24], convolving with time reversal probes is done before any other receiver processing, while in Fig. 4 PPC processing takes place after $\mathrm{CP}$ removal and FFT preprocessing.

Table I shows the the number of complex multiplication $(\times)$, division $(\div)$, addition $(+)$ for the channel estimation and equalisation (in the case of OFDM); and for the PPC operation (in the case of OFDM-PPC). The figures are calculated for the processing of $M+1$ symbols, including one probe/pilot symbol followed by $M$ OFDM symbols. As indicated by the table, the conventional OFDM scheme has a higher computational complexity than the proposed OFDM-PPC scheme. The proposed scheme involves complex multiplication and addition operations; whereas the conventional OFDM system (as shown by (2)) also requires complex divisions. Generally speaking, a complex divider would require 3 times more logic resources than a complex multiplier; a 16-bit complex multiplier takes about three clock cycles to complete, while a complex divider could take up to 20 clock cycles. Therefore, the proposed scheme reduces computational load and latency compared to the conventional OFDM scheme.

The only restriction of the proposed system is that the employed modulation scheme has to be $Q$-ary Phase Shift Keying (PSK) for which amplitude scaling (due to the scaling factor $\left.\left|H_{n}(k)\right|^{2}\right)$ does not affect the symbol decision making process. It is also evident from (4) that the signals from different branches are automatically and constructively added 3 up, consequently, a spatial diversity gain is achieved by 


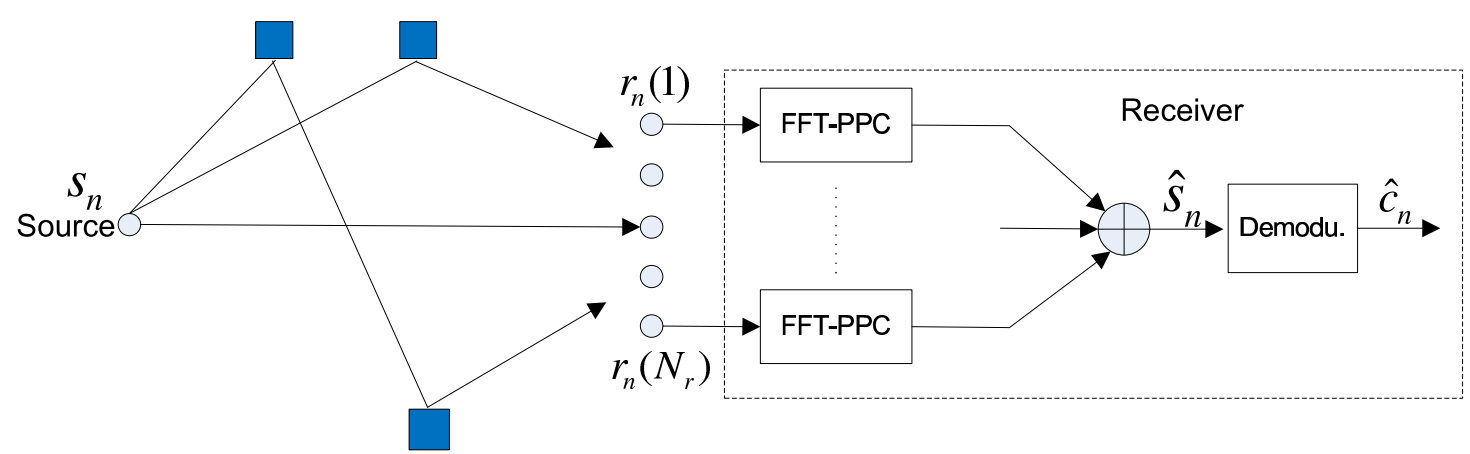

Fig. 3. Proposed OFDM-PPC scheme using a single source and multiple receiver array elements.

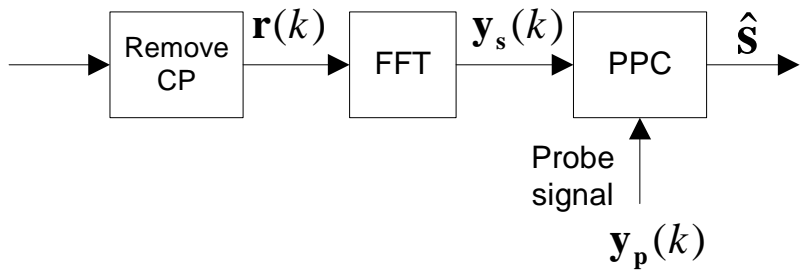

Fig. 4. Block diagram of each individual FFT-PPC branch.

the proposed OFDM-PPC multi-element receiver without additional computational overhead.

When the receiver array elements are used in the conventional OFDM system (shown in Fig. 2), the received signal at the output of the $k$ th array element is formed according to (1) as $y_{n}(k)=s_{n} \cdot H_{n}(k)+\eta_{n}(k)$. To facilitate analysis and to set a benchmark for comparison purposes, let us assume a perfect channel estimate. In this case, the equaliser coefficient becomes [18]

$$
\alpha_{n}(k)= \begin{cases}1 / H_{n}(k), & \text { ZF equaliser } \\ \frac{H_{n}^{*}(k)}{\left|H_{n}\right|^{2}(k)+\sigma_{v}^{2}}, & \text { MMSE equaliser }\end{cases}
$$

and

$$
\begin{aligned}
\hat{s}_{n} & =\sum_{k=1}^{N_{r}} \alpha_{n}(k) y_{n}(k) \\
& =\left\{\begin{array}{l}
N_{r} s_{n}+\sum_{k=1}^{N_{r}} \epsilon_{n}(k), \quad \mathrm{ZF} \\
s_{n} \sum_{k=1}^{N_{r}} \frac{\left|H_{n}(k)\right|^{2}}{\left|H_{n}(k)\right|^{2}+\sigma_{v}^{2}}+\sum_{k=1}^{N_{r}} \varepsilon_{n}(k),
\end{array}\right.
\end{aligned}
$$

Comparing Equation (7) to Equation (4), it is obvious to see that the conventional OFDM does not combine signals as constructively as the proposed OFDM-PPC scheme. There are some other alternatives for estimating symbols, e.g., a least-square solution for channel estimation and equalisation was proposed in [22]. The comparative results presented in the next section shows the performance advantages of the proposed OFDM-PPC over the conventional OFDM.

\section{Comparative Results}

The proposed approach is compared with both the previously reported PPC and the conventional OFDM approaches

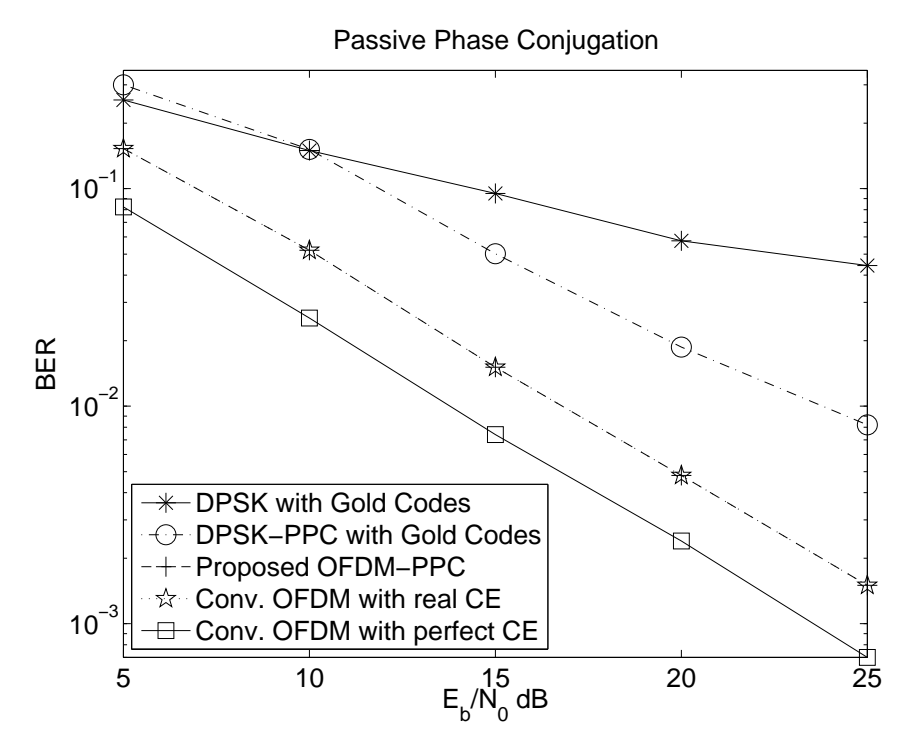

Fig. 5. Simulated performance comparison between different systems. The number of array element $N_{r}=1$.

in this section. For simulation of the multipath channels, we adopt the SUI-3 channel model used for the $802.16 \mathrm{~d}$ fixed WiMAX systems [26]. This is a slowly time-varying channel for which the channel reciprocity property holds and the correlate-and-sum principle of PPC is applicable. The channel has a tap spacing of 500ns, and maximum tap delay of 1000ns. For the PPC system proposed by Hursky, et al. [6], we use DPSK modulation and Gold codes of length 31 , the data rate is $64.5 \mathrm{Kbps}$; for the OFDM based systems, the modulation scheme is QPSK, the FFT size is chosen to be $N=64$, the length of $\mathrm{CP}$ is 4 , the data rate is 4 Mbps. A block-type least square channel estimation scheme is employed for the OFDM systems. In this case, all subcarriers are used as pilots during the training period. The simulation curves are obtained by averaging the simulation results over a minimum of 1000 blocks of transmitted data and after at least 100 errors have occurred.

\section{A. Single Receiver}

Fig. 5 shows that in a single input single output situation, the proposed OFDM-PPC system is superior to the PPC 
system in terms of bit error rate (BER) performance. Note that DPSK with PPC performs better than DPSK without PPC because PPC can focus the multipath arrivals and remove phase ambiguity. In Fig. 5 we also compare the proposed OFDM-PPC with the conventional OFDM system with i) imperfect channel estimate (real CE); ii) perfect knowledge of the channel information (perfect $\mathrm{CE}$ ). In the first case, we estimate the channel with pilots; in the latter case, we assume the channel frequency response $H_{n}$ is perfectly known to the receiver, this is an idealized situation and serves as a performance lower bound for the OFDM system.

Fig. 5 shows that the performance of the proposed system coincides with that of the conventional OFDM system employing MMSE equaliser with real CE. However, this is achieved at a lower complexity since channel estimation and equalisation are no longer needed. In addition, the complex multiplication involved in PPC is much easier to implement than the complex division involved in equalisation.

For the proposed OFDM-PPC, there is a spectral efficiency (transmission rate) loss of $1 / M$ (where $M$ stands for the number of OFDM symbols transmitted after each probe) due to the probe transmission, but it should be noted that the conventional OFDM systems also need pilots for channel estimation. One can also observe from Fig. 5 that both systems are $3 \mathrm{~dB}$ above the performance lower bound obtained by assuming perfect channel estimates.

In IEEE 802.11a [19], each OFDM symbol has a duration of $4 \mu s(0.8 \mu s$ for $\mathrm{CP}$ and $3.2 \mu s$ for data). Channel estimation is carried out for each burst which has a duration of $4 \mathrm{~ms}$ for indoor environment [19]. In this case, $M=1000$, i.e., only one probe is needed for every 1000 OFDM symbols. In the LTE radio standard [21] which is mostly concerned with mobile environments, channel coherence time is equal to 9 $\mathrm{ms}$ at a vehicle speed of $30 \mathrm{~km} / \mathrm{h}$. Each LTE frame has $10 \mathrm{~ms}$ in duration, and is divided into 10 subframes, each of which is further divided into two $0.5 \mathrm{~ms}$ slots. One slot consists of either 6 or 7 OFDM symbols, depending on whether the normal or extended cyclic prefix is employed [21]. In this case, $M \approx 100$, i.e., only one probe is needed for every 100 OFDM symbols. Consequently, in either of these cases a spectrum loss of $1 / M=0.01$ or 0.001 is not significant.

\section{B. Multiple Receivers}

The results shown in Fig. 5 are obtained for systems with single array element. In the following simulations, we consider the case when the number of array elements $N_{r}>1$. The array diversity gain is examined in Fig. 6 where we plot the results corresponding to $N_{r}=2,4$, respectively. The MMSE equaliser is used for the conventional OFDM system. Here it can be seen that the performances of all the systems improve significantly as the number of array elements $N_{r}$ increases. When $N_{r}>1$, the proposed scheme outperforms the conventional OFDM with real channel estimate and converges to the conventional OFDM with perfect channel estimate at high SNR. This is due to the fact that i) the antenna array placed at receiver contributes to better temporal focusing as shown in [25]; ii) the proposed OFDM-PPC

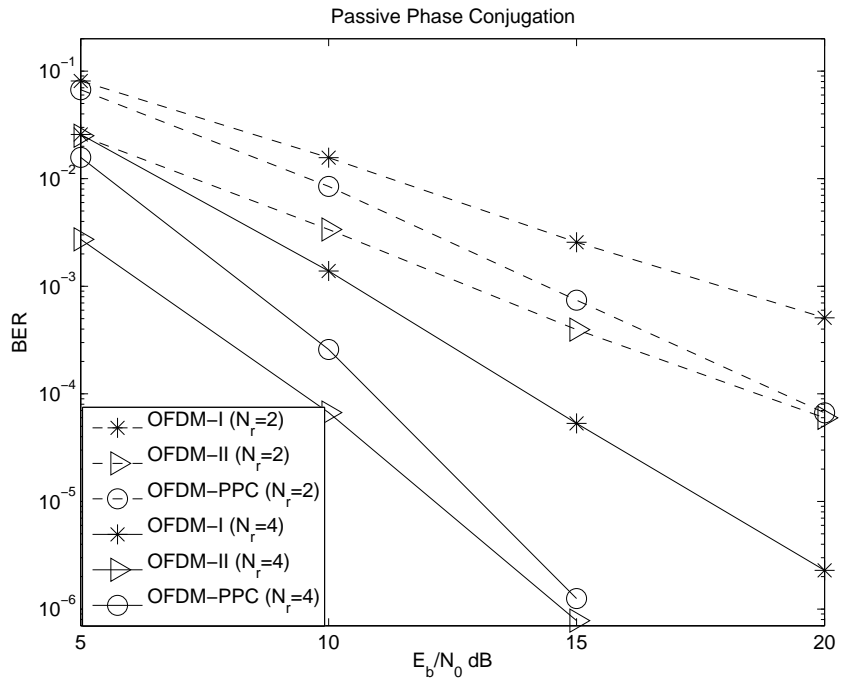

Fig. 6. Performance comparison with different number of receiver array elements. OFDM-I stands for the conventional OFDM with real CE; OFDMII stands for the conventional OFDM with perfect CE.

scheme achieves array diversity gain by optimum combining and automatic constructive signals summation from different array elements, which is not the case for the conventional OFDM system shown by (7).

\section{Multiple Receivers with Mutual Coupling}

In real propagation environments, source to receiver array channels are correlated to a certain extent due to their finite separation, geometry of array and effect of surrounding objects in the near field of the receiving antenna elements [27]. For the purpose of evaluating the effect that mutual coupling between receiver elements has on OFDM-PPC, the mutual coupling is defined, after [26], as the envelope correlation coefficient between signals received at each array element, i.e.,

$$
\begin{aligned}
\rho & =\left|\frac{\mathrm{E}\left\{\left(h_{i}(l)-E\left\{h_{i}(l)\right\}\right)\left(h_{m}(l)-E\left\{h_{m}(l)\right\}\right)^{*}\right\}}{\sqrt{\mathrm{E}\left\{\left|h_{i}(l)-E\left\{h_{i}(l)\right\}\right|^{2}\right\} \mathrm{E}\left\{\left|h_{m}(l)-E\left\{h_{m}(l)\right\}\right|^{2}\right\}}}\right| \\
& =\left|\frac{\operatorname{cov}\left(h_{i}(l), h_{m}(l)\right)}{\sqrt{\operatorname{var}\left(h_{i}(l)\right) \operatorname{var}\left(h_{m}(l)\right)}}\right|,
\end{aligned}
$$

where $h_{i}(l) / h_{m}(l)$ denotes the channel coefficient corresponding to the $l$ th tap of the impulse response of the channel between the source and the $i \mathrm{th} / m$ th receiver array element.

In Fig. 7, we show the impact of mutual coupling on the performance of different systems. Here the number of array element $N_{r}=2$, and the correlation factor $\rho$ is set to be 0.2 . Compared to the case without mutual coupling (as shown in Fig. 6), moderate mutual coupling $(\rho=0.2)$ does not have a significant effect on all the simulated scenarios.

The impact of the mutual coupling is further examined for the proposed OFDM-PPC scheme in Fig. 8 for $N_{r}=$ $2, N_{r}=4$, respectively. It is evident from the figure that the array diversity gradually diminishes as the correlation factor 5 


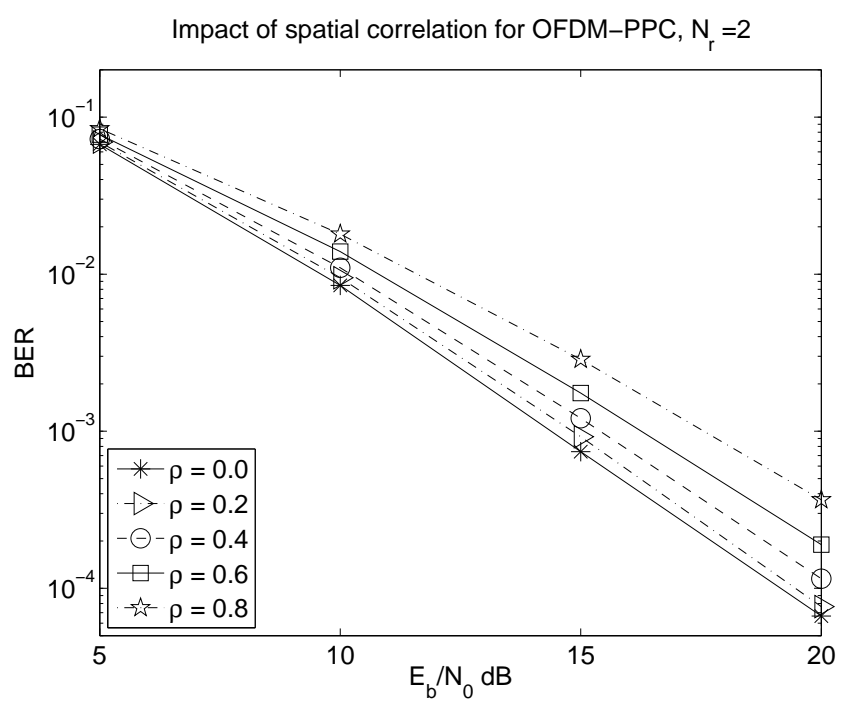

(a) $N_{r}=2$.

Fig. 8. Impact of the mutual coupling on the proposed OFDM-PPC.

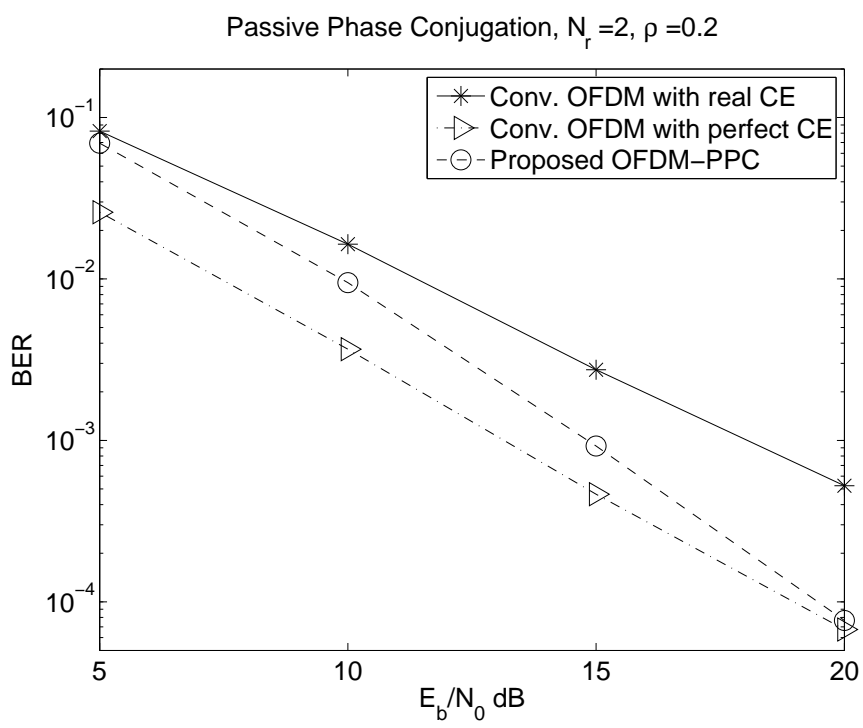

Fig. 7. Impact of mutual coupling $\left(N_{r}=2\right)$

(e.g., $\rho=0.2$ ) do not have noticeable effect on system performance. As shown in [28], the value of $\rho$ for practical half-wave dipole antennas is in the range of 0.2 and 0.3 for the antenna separation of half a wavelength. Therefore mutual coupling does not impose a big concern for the application of the proposed OFDM-PPC scheme to practical systems. Comparing Fig. 8(a) with Fig. 8(b), one can see that mutual coupling has a greater effect for systems with more array elements.

Fig. 9 shows the impact of channel mismatch on the performance of the conventional OFDM and the proposed OFDM-PPC system. The channel mismatch is simulated by a random process with zero mean and variance $\sigma_{e}^{2}$. The number of receiver antennas is $N_{r}=2$ and the SNR value

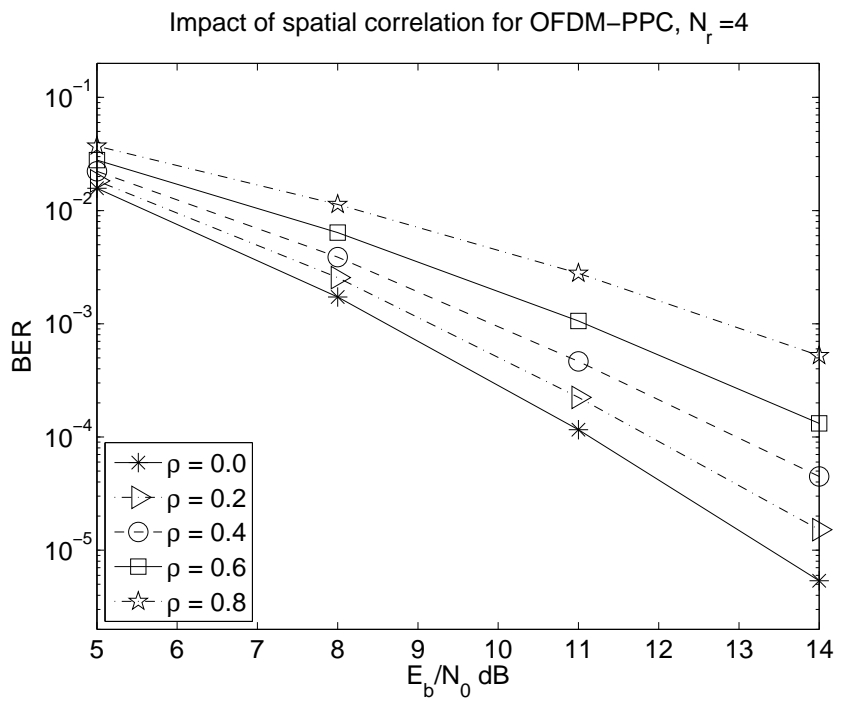

(b) $N_{r}=4$.

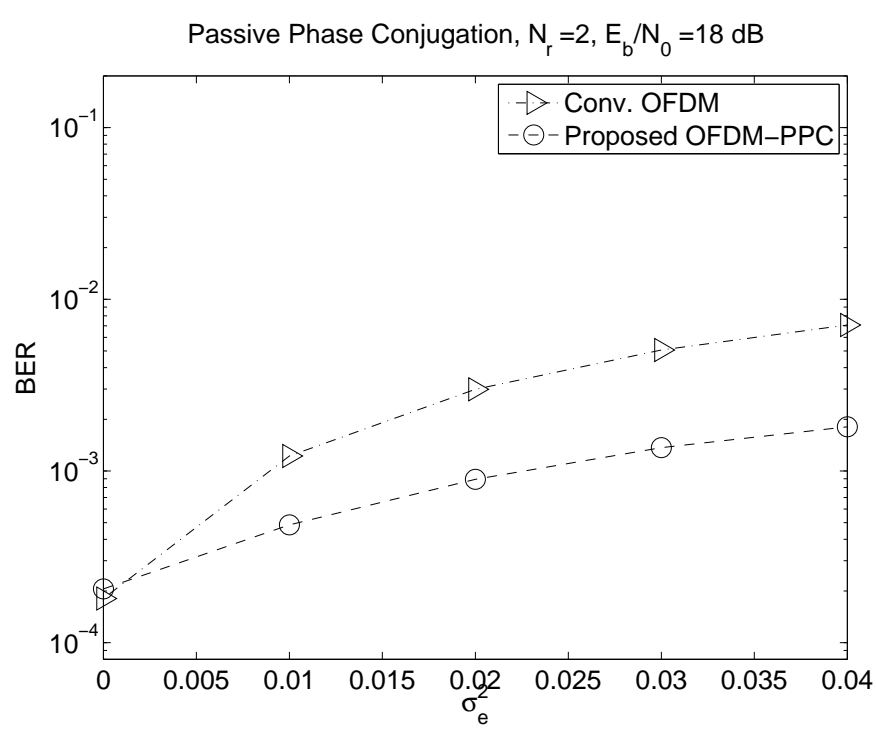

Fig. 9. Impact of channel mismatch on the performance of OFDM-PPC and OFDM.

is $E_{b} / N_{0}=18 \mathrm{~dB}$. One can see from the figure that the performance of both systems degrades as the degree of mismatch increases, and the conventional OFDM is more sensitive to channel mismatch than the proposed OFDM-PPC.

In Fig. 10, we compare two systems with error control coding. In this case, the FFT size is chosen to be 512 , the employed convolution code has a rate of $1 / 2$, constraint length of 7 and generator polynomial $(133,171)$ in octal form. We use a random interleaver, and the interleaver length is 1024 . The soft symbol-to-bit demapper is implemented by extracting the in-phase and quadrature components of the PPC output (in the case of the proposed OFDM-PPC) or the equalizer output (in the case of the conventional OFDM) [29]. The 


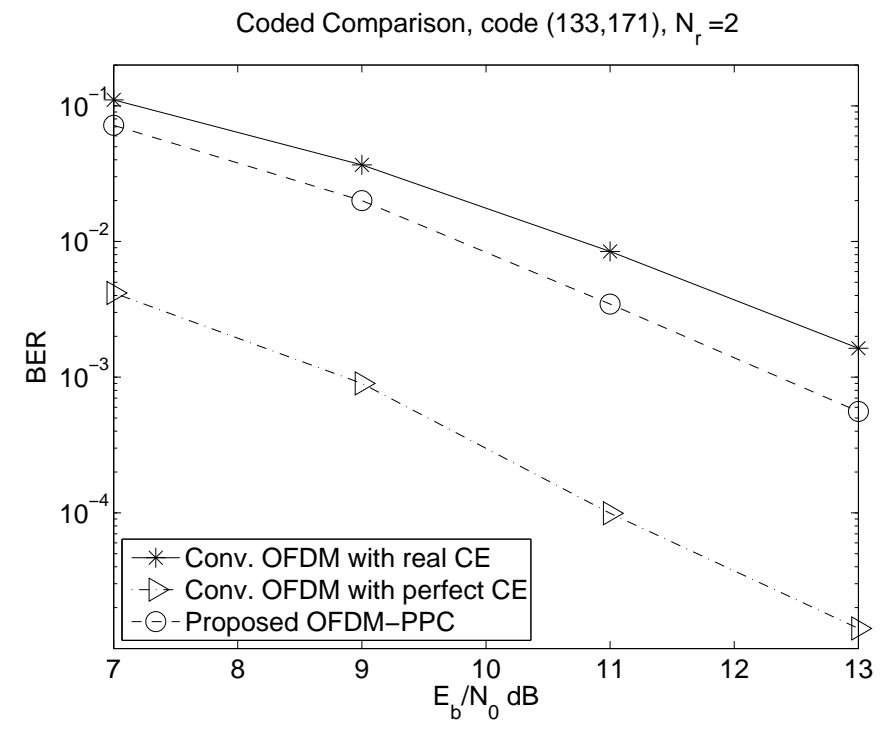

Fig. 10. Comparison between OFDM and OFDM-PPC in coded systems.

soft bit estimates are deinterleaved and passed to the Viterbi decoder to perform soft-input Viterbi decoding. Comparing Fig. 10 with Fig. 6, it is evident to see that employing error control coding enables an OFDM system to exploit frequency diversity gain and achieve much improved performance. In the coded case, the proposed OFDM-PPC still performs better than the conventional OFDM with real $\mathrm{CE}$, a performance of gain of $1 \mathrm{~dB}$ has been observed.

Comparing Fig. 10 with Fig. 6, one can also conclude that it is more advantageous to apply the proposed scheme in uncoded systems. Such a scenario arises, e.g., for Gigabit/sec communications at millimeter wave frequencies [30] where uncoded transmission in a multipath rich environment occurs.

\section{THEORETICAL ANALYSIS OF OFDM-PPC FOR MULTIPATH CHANNELS WITH UNIFORM PDP}

In this section, we derive a closed form expression to predict the average BER performance of the proposed OFDMPPC scheme with multiple array elements in order to provide a theoretical foundation for our earlier simulated findings. For simplicity, our analysis is conducted for multipath channels with uniform power delay profile (PDP) and without mutual coupling.

Assuming the fading is identically distributed with the same fading parameter, we have $\gamma_{k}=\mathrm{E}\left[\left|H_{n}(k)\right|^{2}\right]=\gamma$ for all $k=1, \ldots, N_{r}$. A closed-form expression for the average bit error probability for the OFDM-PPC system can be written as

$$
P_{b}=\frac{1}{2}\left[1-\sum_{k=0}^{N_{r}-1} \mu\left(\frac{1-\mu^{2}}{4}\right)^{k}\left(\begin{array}{c}
2 k \\
k
\end{array}\right)\right],
$$

where

$$
\mu=\sqrt{\frac{E_{b} \gamma}{E_{b} \gamma+2 E_{b} N_{0}+\left|p_{n}\right|^{2} N_{0}}} .
$$

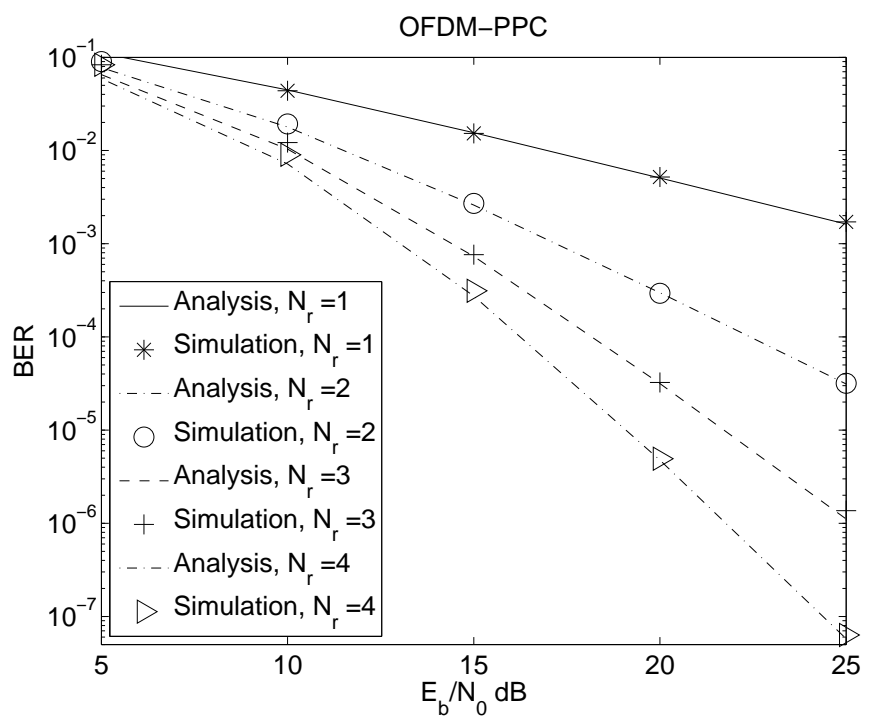

Fig. 11. Analysis vs. simulation. OFDM-PPC for multipath channels with uniform power delay profile. The number of receive antennas $N_{r}=$ $1,2,3,4 ; \rho=0$.

Refer to appendix for a detailed derivation of the above formula.

The analytical results derived by Eq. (9) for different numbers of array elements are compared with the simulation results in Fig. 11. Here the FFT size is 512, the length of cyclic prefix is 16, the channel has 13 taps with uniform PDP. It can be seen from Fig. 11 that the analytical results are in close agreement with the simulation results at medium to high SNRs, slight disrepancies are observed at low SNR, which indicates the approximation made in Eq. (18) is valid, and the cross-noise variance $N_{0}^{2}$ can be safely ignored at high SNRs. The comparative results verify our theoretical analysis, and provide further insights into the behavior of OFDM-PPC and prediction of its BER performance.

Fig. 12 shows the predicted (by Eq. (9)) performance of the proposed OFDM-PPC for different number of array elements. These results suggest that BER of $10^{-6}$ can be achieved at $E_{b} / N_{0}$ of $20 \mathrm{~dB}$ for $N_{r}=5$ in the proposed OFDMPPC system without channel coding. However, in practical systems, channel mismatch will degrade system performance as illustrated in Fig. 9.

\section{CONCLUSIONS}

The OFDM technique is widely used in practical radio communication systems to combat frequency selective fading. On the other hand, PPC has been adopted within the underwater acoustic communications community in order to constructively combine multipath arrivals. We have introduced a hybrid OFDM-PPC scheme which utilizes the desired properties of both OFDM and PPC, and requires only minor modification to the classic OFDM system. The results we obtained show that the proposed scheme outperforms the conventional OFDM while preserving reduced computational 


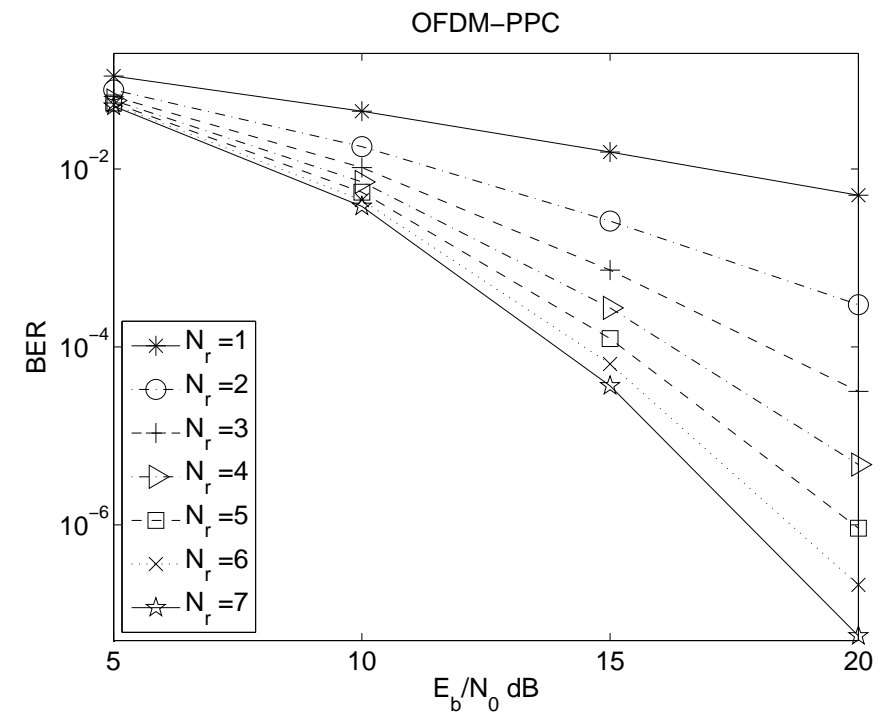

Fig. 12. Predicted performance of the proposed OFDM-PPC with different number of array elements $N_{r} \cdot \rho=0$.

advantageous in systems with multiple receiver array elements by facilitating automatic and constructive combining of the signalsreceived. The work reported here should find applications in situations where the propagation environment cannot be accurately estimated and where data transmission rate is premium.

\section{ACKNOWLEDGEMENT}

The work presented in this paper has been supported by the Department of Education and Learning for Northern Ireland, under the Strengthening All Island (SAI) - Mobile Wireless Future project. An UK patent "OFDM with PPC" (with reference number GB10156156) related to this work has been filed on September 10, 2010.

\section{APPENDIX}

Based on (5), we can derive the variance of the Gaussian random variable $\omega_{n}(k)$ as $\omega_{n}(k)$ as

$$
\begin{aligned}
N_{\omega_{n}(k)}= & \mathrm{E}\left[\left|\omega_{n}(k)\right|^{2}\right]=\left|H_{n}(k)\right|^{2} E_{s} \mathrm{E}\left[\left|\epsilon_{n}(k)\right|^{2}\right] \\
& +\left|H_{n}\right|^{2}\left|p_{n}\right|^{2} \mathrm{E}\left[\left|\xi_{n}(k)\right|^{2}\right]+N_{0}^{2} \\
= & \left|H_{n}(k)\right|^{2} E_{s} N_{0}+\left|H_{n}(k)\right|^{2}\left|p_{n}\right|^{2} N_{0}+N_{0}^{2} \\
= & \left|H_{n}(k)\right|^{2} N_{0}\left(E_{s}+\left|p_{n}\right|^{2}\right)+N_{0}^{2},
\end{aligned}
$$

where $E_{s}=\mathrm{E}\left[\left|s_{n}\right|^{2}\right]$ is the average symbol energy. The channel frequency response at the $n$th subcarrier is given by the $n$th discrete Fourier transform (DFT) coefficient of the channel impulse response. According to the DFT definition, we have

$$
H_{n}(k)=\sum_{l=0}^{L-1} h_{l}(k) \exp \left(-j \frac{2 \pi}{N} n l\right),
$$

where $h_{l}(k)$ is the $l$ th channel coefficient from the transmitter to the $k$ th receiver array element, assumed to be zero mean complex Gaussian random variable with variance $P_{l}(k)=$ $\mathrm{E}\left[\left|h_{l}(k)\right|^{2}\right]$.

Thus, the Gaussian random variable $h_{l}(k)$ can be denoted as

$$
h_{l}(k) \sim \mathcal{C N}\left(0, P_{l}(k)\right) .
$$

Since $h_{l}(k)$ is a complex Gaussian random variable, $H_{n}(k)=\sum_{l=0}^{L-1} h_{l}(k) \exp \left(-j \frac{2 \pi}{N} n l\right)$ is also a complex Gaussian random variable. Based on Eq. (11), its variance can be computed as

$$
\begin{aligned}
\mathrm{E}\left[\left|H_{n}(k)\right|^{2}\right] & =\sum_{l=0}^{L-1} \mathrm{E}\left[\left|h_{l}(k) \exp \left(-j \frac{2 \pi}{N} n l\right)\right|^{2}\right] \\
& =\sum_{l=0}^{L-1} \mathrm{E}\left[\left|h_{l}(k)\right|^{2}\right]=\sum_{l=0}^{L-1} P_{l}(k) .
\end{aligned}
$$

The above equation holds since $|\exp (j \omega)|^{2}=1$ for any value of $\omega$. We can now denote $H_{n}(k)$ as

$$
H_{n}(k) \sim \mathcal{C N}\left(0, \sum_{l=0}^{L-1} P_{l}(k)\right) .
$$

Without loss of generality, we assume the variance of $H_{n}(k)$ is normalized such that

$$
\mathrm{E}\left[\left|H_{n}(k)\right|^{2}\right]=\gamma_{k}=\sum_{l=0}^{L-1} P_{l}(k)=1 / N_{r} .
$$

In the case when the probe is chosen to be an all-one sequence, i.e., $\mathbf{p}=\left[\begin{array}{llll}1 & 1 & \ldots & 1\end{array}\right]$, (4) simplifies to

$$
\mathbf{z}=\left[\begin{array}{c}
s_{1} \sum_{k=1}^{N_{r}}\left|H_{1}(k)\right|^{2}+\omega_{1} \\
s_{2} \sum_{k=1}^{N_{r}}\left|H_{2}(k)\right|^{2}+\omega_{2} \\
\vdots \\
s_{N} \sum_{k=1}^{N_{r}}\left|H_{N}(k)\right|^{2}+\omega_{N}
\end{array}\right]^{T} .
$$

The $n$th entry in (12) represents the decision statistics for the $n$th symbol, it can be expressed as

$$
z_{n}=s_{n} \sum_{k=1}^{N_{r}}\left|H_{n}(k)\right|^{2}+\sum_{k=1}^{N_{r}} \omega_{n}(k)=s_{n} G_{n}+w_{n},
$$

where $G_{n}=\sum_{k=1}^{N_{r}}\left|H_{n}(k)\right|^{2}$, and $w_{n}=\sum_{k=1}^{N_{r}} \omega_{n}(k) \sim$ $\mathcal{C N}\left(0, N_{w}\right)$. Based on (10), the variance of $w_{n}$ is

$$
\begin{aligned}
N_{w} & =\mathrm{E}\left[\left|w_{n}\right|^{2}\right]=\sum_{k=1}^{N_{r}} N_{\omega_{n}(k)} \\
& =\left(E_{s}+\left|p_{n}\right|^{2}\right) N_{0} \sum_{k=1}^{N_{r}}\left|H_{n}(k)\right|^{2}+N_{r} N_{0}^{2} \\
& =\left(E_{s}+\left|p_{n}\right|^{2}\right) N_{0} G_{n}+N_{r} N_{0}^{2} .
\end{aligned}
$$

The signal-to-noise ratio (SNR) for the decision statistic $z_{n}$ in (13) can be calculated as

$$
\begin{aligned}
S N R & =\frac{\mathrm{E}\left[\left|s_{n} G_{n}\right|^{2}\right]}{\mathrm{E}\left[\left|w_{n}\right|^{2}\right]}=\frac{G_{n}^{2} E_{s}}{N_{w}} \\
& =\frac{G_{n}^{2} E_{s}}{\left(E_{s}+\left|p_{n}\right|^{2}\right) N_{0} G_{n}+N_{r} N_{0}^{2}} .
\end{aligned}
$$


In the case of QPSK modulation, one symbol corresponds to 2 bits, the relation between symbol energy and bit energy is $E_{s}=2 E_{b}$, therefore

$$
S N R=\frac{2 G_{n}^{2} E_{b}}{\left(2 E_{b}+\left|p_{n}\right|^{2}\right) G_{n} N_{0}+N_{r} N_{0}^{2}} .
$$

The bit error probability (BER) denoted as $P_{b}$ is uniquely determined by SNR, more specifically, $P_{b}=Q(\sqrt{S N R})$, where $Q(x)$ is the complementary Gaussian cumulative distribution function [31]. Therefore, the bit error probability conditioned on the random variable $G_{n}$ can be expressed as

$$
\begin{aligned}
P_{b}\left(G_{n}\right) & =Q(\sqrt{S N R}) \\
& =Q\left(\sqrt{\frac{2 G_{n}^{2} E_{b}}{\left(2 E_{b}+\left|p_{n}\right|^{2}\right) G_{n} N_{0}+N_{r} N_{0}^{2}}}\right) .
\end{aligned}
$$

In order to obtain the average bit error probability, we need to average $P_{b}\left(G_{n}\right)$ over the distribution of the random variable $G_{n}=\sum_{k=1}^{N_{r}}\left|H_{n}(k)\right|^{2}$, i.e.,

$$
\begin{aligned}
P_{b} & =\int_{0}^{\infty} P_{b}\left(G_{n}\right) f\left(G_{n}\right) d G_{n} \\
& =\int_{0}^{\infty} Q\left(\sqrt{\frac{2 G_{n}^{2} E_{b}}{\left(2 E_{b}+\left|p_{n}\right|^{2}\right) G_{n} N_{0}+N_{r} N_{0}^{2}}}\right) f\left(G_{n}\right) d G_{n},
\end{aligned}
$$

where $f\left(G_{n}\right)$ is the probability density function (PDF) of $G_{n}$. Since $H_{n}(k)$ is a Gaussian random variable, $\left|H_{n}(k)\right|^{2}$ is a Chi-square random variable. Considering the multipath channel with a uniform power delay profile (PDP), i.e., the fading is assumed to be identically distributed with the same fading parameter, we have $\gamma_{k}=\mathrm{E}\left[\left|H_{n}(k)\right|^{2}\right]=\gamma$ for all $k$. The PDF of $G_{n}$ can be derived as [31]

$$
f\left(G_{n}\right)=\frac{1}{\gamma^{N_{r}}\left(N_{r}-1\right) !} G_{n}^{N_{r}-1} \exp \left(-G_{n} / \gamma\right), \quad G_{n} \geq 0 .
$$

Substituting (16) into (14) yields

$$
\begin{aligned}
& P_{b}=\int_{0}^{\infty} P_{b}\left(G_{n}\right) f\left(G_{n}\right) d G_{n}=\frac{1}{\gamma^{N_{r}}\left(N_{r}-1\right) !} \\
& \underbrace{\int_{0}^{\infty} Q\left(\sqrt{\frac{2 G_{n}^{2} E_{b}}{\left(2 E_{b}+\left|p_{n}\right|^{2}\right) G_{n} N_{0}+N_{r} N_{0}^{2}}}\right) G_{n}^{N_{r}-1} e^{-G_{n} / \gamma} d G}_{\Phi^{\prime}}
\end{aligned}
$$

A closed-form expression for integral $\Phi^{\prime}$ in (17) is difficult to derive. The derivation can be simplified if the cross-noise variance $N_{0}^{2}$ in the denominator of the Q-function is omitted, which is a valid approximation at high SNR when $N_{0}$ is small. In this case, Eq. (17) can be approximated as

$$
\begin{aligned}
& P_{b} \approx \frac{1}{\gamma^{N_{r}}\left(N_{r}-1\right) !} \\
& \cdot \underbrace{\int_{0}^{\infty} Q\left(\sqrt{\frac{2 G_{n} E_{b}}{\left(2 E_{b}+\left|p_{n}\right|^{2}\right) N_{0}}}\right) G_{n}^{N_{r}-1} e^{-G_{n} / \gamma} d G_{n}}_{\Phi} .
\end{aligned}
$$

The integral $\Phi$ in (18) can be solved by using the following equation [32]:

$$
\begin{aligned}
& \int_{0}^{\infty} Q(\sqrt{a x}) x^{L-1} e^{-x / \gamma} d x \\
& =\frac{1}{2} \gamma^{L} \Gamma(L)\left[1-\sum_{k=0}^{L-1} \mu\left(\frac{1-\mu^{2}}{4}\right)^{k}\left(\begin{array}{c}
2 k \\
k
\end{array}\right)\right],
\end{aligned}
$$

where $\mu=\sqrt{\frac{a \gamma}{2+a \gamma}}$ and $\Gamma(x)$ is the Gamma function [31]. If $x$ is a positive integer, $\Gamma(x)=(x-1)$ !. Assigning $a=$ $\frac{2 E_{b}}{\left(2 E_{b}+\left|p_{n}\right|^{2}\right) N_{0}}$, the integral in (18) can be calculated as

$$
\Phi=\frac{1}{2} \gamma^{N_{r}}\left(N_{r}-1\right) !\left[1-\sum_{k=0}^{N_{r}-1} \mu\left(\frac{1-\mu^{2}}{4}\right)^{k}\left(\begin{array}{c}
2 k \\
k
\end{array}\right)\right] .
$$

Denoting

$$
\mu=\sqrt{\frac{a \gamma}{2+a \gamma}}=\sqrt{\frac{E_{b} \gamma}{E_{b} \gamma+2 E_{b} N_{0}+\left|p_{n}\right|^{2} N_{0}}},
$$

the closed-form expression for the average bit error probability for the OFDM-PPC system can be written as

$$
P_{b}=\frac{1}{\gamma^{N_{r}}\left(N_{r}-1\right) !} \Phi=\frac{1}{2}\left[1-\sum_{k=0}^{N_{r}-1} \mu\left(\frac{1-\mu^{2}}{4}\right)^{k}\left(\begin{array}{c}
2 k \\
k
\end{array}\right)\right] \text {. }
$$

\section{REFERENCES}

[1] M. Stojanovic, J. Catipovic, J. Proakis. "Phase-coherent digital communications for underwater acoustic channels". IEEE Journal of Oceanic Engineering, vol. 19, pp. 100-111, January 1994.

[2] M. Stojanovic. "Recent advances in high-speed underwater acoustic communication". IEEE Journal of Oceanic Engineering, vol. 21, pp. 125-136, April 1996.

[3] F. Fu, D. Doonan, C. Utley, H. Lee. "Field testing of a spread spectrum acoustic modem with sparse channel estimation". Proc. IEEE International Conference on Acoustics, Speech and Signal Processing, pp. 5292-5295, April 2008.

[4] D. Rouseff, D. Jackson, W. Fox, C. Jones, J. Ritcey, D. Dowling. "Underwater acoustic communication by passive-phase conjugation: theory and experimental results". IEEE Journal of Oceanic Engineering, vol. 26, no. 4, pp. 821-831, 2001.

[5] D. Dowling. "Acoustic pulse compression using passive phase-conjugate processing", Journal of the Acoustical Society of America, vol. 95, no. 3, pp. 1450-1458, March 1994.

[6] P. Hursky, M. Porter, M. Siderius. "Point-to-point underwater acoustic communications using spread-spectrum passive phase conjugation", Journal of the Acoustical Society of America, vol. 120, no. 1, pp. 247257, July 2006.

[7] J. Flynn, J. Ritcey, D. Rouseff, W. Fox. "Multichannel equalization by decision-directed passive phase conjugation: experimental results". IEEE Journal Oceanic Engineering, vol. 29, no. 3, pp. 824-836, July 2004.

[8] J. Gomes, V. Barroso. "Asymmetric underwater acoustic communication using time-reversal mirror". Proc. IEEE Oceans'01 Conference, vol. 3, pp. 1847-1851, Sept. 2000.

[9] J. Gomes, V. Barroso. "Ray-based analysis of a time-reversal mirror for underwater acoustic communication". Proc. IEEE ICASSP'OO Conference, pp. 2981-2984, June 2000.

[10] G. Edelmann, T. William, S. Hodgekiss, S. Kim, W. Kuperman, H. Song. "An initial demonstration of underwater acoustic communication using time reversal", IEEE Journal on Oceanic Engineering, vol. 27, no. 3, 602-609, July 2002.

[11] W. Kuperman, W. Hodgkiss, H. Song. "Phase conjugation in the ocean: experimental demonstration of an acoustic time-reversal mirror", Journal of the Acoustical Society of America, vol. 103, no. 1, pp. 25-40, 
[12] T. Yang. "Temporal resolutions of time-reversed and passive-phase conjugation for underwater acoustic channel", IEEE Journal Oceanic Engineering, vol. 28, no. 2, pp. 229-245, 2003.

[13] H. Song, P. Roux, W. Hodgkiss, W. Kuperman, T. Akal, M. Stevenson. "Multiple-input/multiple-output coherent time reversal communications in a shallow water acoustic channel", IEEE Journal Oceanic Engineering, vol. 31, no. 1, pp. 170-178, Jan. 2006.

[14] H. Song, W. Hodgkiss, W. Kuperman. "MIMO time reversal communications", Proc. WuWNet, pp. 5-10, Sept. 14, 2007.

[15] J. Tuovinen, G. Shiroma, W. Forsyth, W. Shiroma. "Multipath communications using a phase-conjugate array", IEEE MTT-S International Microwave Symposium, vol. 3, pp. 1681-1684, June 2003.

[16] B. Henty, D. Stancil. "Multipath-enabled super-resolution for RF and microwave communication using phase-conjugate arrays", Physical Review Letters, vol. 93, no. 24, 243904, Dec. 2004.

[17] M. Stojanovic. "Retrofocusing techniques for high rate acoustic communications". Journal of the Acoustical Society of America, vol. 117, no. 3, pp. 1173-1185, March 2005

[18] H. Sari, G. Karam, I. Jeanclaude. "Transmission techniques for digital terrestrial TV broadcasting”. IEEE Communications Magazine, pp. 100109, Feb. 1995.

[19] IEEE Std 802.11a-1999, Part 11: Wireless LAN Medium Access Control (MAC) and Physical Layer (PHY) Specifications - High-speed Physical Layer in the $5 \mathrm{GHz}$ Band, IEEE, 1999.

[20] 3GPP TR 25.814 V7.1.0. Physical layer aspects for evolved UTRA Tech. Rep., 3GPP Organizational Partners, Sophia Antipolis, France, Sept. 2006. Available at http://www.quintillion.co.jp/3GPP/Specs/25814710.pdf.

[21] J. Zyren, W. Mccoy. Overview of the 3GPP Long Term Evolution Physical Layer. White Paper, Freescale Semiconductor Inc., Arizona, USA, July 2007

22] B. Li, S. Zhou, M. Stojanovic, L. Freitag, P. Willett. "Multicarrie communication over underwater acoustic channels with nonuniform Doppler shifts", IEEE Journal of Oceanic Engineering, vol. 33, no. 2, pp. 198-209, April 2008.

[23] J. Gomes, A. Silva, S. Jesus. "Experimental assessment of timereversed OFDM underwater communications". The Journal of the Acoustical Society of America, vol. 123, no. 5, pp. 3891, May 2008.

[24] J. Gomes, A. Silva, S. Jesus. "OFDM demodulation in underwater time-reversed shortened channels.". Proc. IEEE OCEANS'08, Canada, Sept. 2008.

[25] P. Xiao, V. Fusco, P. Sundaralingam. "Analysis of temporal compression characteristics using active phase conjugation in systems with multiple antenna elements", Proc. EuCAP, pp. 297-300, April 2011.

[26] V. Erceg et al. "Channel models for fixed wireless applications". IEEE 802.16a cont. IEEE 802.16.3c-01/29r4, June 2003.

[27] M. K. Ozdemir, E. Arvas. "Dynamics of spatial correlation and implications on MIMO systems". IEEE Communications Magazine, vol 42, no. 6, pp. 514-519, June 2004.

[28] A. Derneryd, G. Kristensson. "Signal correlation including antenna coupling”. IET Electronic Letters, vol. 40, no. 3, pp. 157-159, Feb. 2004.

[29] F. Tosato, P. Bisaglia. "Simplified soft-output demapper for binary interleaved COFDM with application to HIPERLAN/2". Proc. IEEE ICC, vol. 2, pp. 664-668, 2002.

[30] IEEE 802.15 WPAN, Task Group 3c, Millimeter Wave Alternative PHY. Part 15.3: Wireless Medium Access Control (MAC) and Physical Layer (PHY) Specifications for High Rate Wireless Personal Area Networks (WPANs), IEEE, 3 Park Avenue, New York, NY 10016-5997, USA, Oct. 2009.

[31] J. Proakis, Digital Communications. 3rd edition, McGraw-Hill, 1995.

[32] H. Zhang, T. Gulliver. "Error probability for maximum ratio combining multichannel reception of $\mathrm{M}$-ary coherent systems over flat ricean fading channels". In Proc. IEEE Wireless Communications and Networking Conference (WCNC'04), vol. 1, Atlanta, GA, USA, Mar. 2004, pp. 306310 . 